\title{
ANÁLISE DA UTILIZAÇÃO DAS FERRAMENTAS GERENCIAIS NA CONSTRUÇÃO CIVIL ENTRE MPEs E MGES
}

\section{ANALYSIS OF THE USE OF MANAGEMENT TOOLS IN CIVIL CONSTRUCTION BETWEEN SMES AND MLES}

\section{AMANDA PATRÍCIA MACIEL}

Universidade Tecnológica Federal do Paraná - UTFPR

Especialista em Gerenciamento de Obras

Orcid: https://orcid.org/0000-0003-3341-0455 / E-mail: aamanda.maciel@hotmail.com Rua Jovino Cavalheiro dos Santos, 128 - Atuba, Curitiba - PR, 82630-305.

\section{ALFREDO IAROZINSKI NETO}

Universidade Tecnológica Federal do Paraná - UTFPR

Doutorado em Engenharia

Orcid: https://orcid.org/0000-0002-3160-5251 / E-mail: alfredo.iarozinski@gmail.com

Submissão: 02/07/2021. Revisão: 24/09/2021. Aceite: 08/10/2021. Publicação: 01/02/2022. DOI: http://dx.doi.org/10.22277/rgo.v15i1.6538

\section{RESUMO}

Objetivo: O objetivo deste trabalho é identificar quais ferramentas gerenciais diferenciam as micro e pequenas empresas das médias e grandes organizações, bem como avaliar o nível de utilização dessas ferramentas, levando em consideração o tamanho das organizações.

Método / abordagem: A pesquisa foi baseada em um Survey, que através de um questionário, levantou dados de 89 empresas da construção civil situadas na Região Metropolitana de Curitiba. Foi realizada uma comparação, por meio da análise discriminante e análise descritiva dos dados coletados, quanto à utilização das técnicas gerenciais entre micro e pequenas empresas e médias e grandes organizações.

Principais resultados: Os resultados mostraram que a certificação do Programa Brasileiro da Qualidade e Produtividade do Habitat e o sistema de custeio $A B C$ são os métodos que mais diferenciam as grandes e médias organizações das micro e pequenas empresas. Também demonstraram que as ferramentas de gestão de obras são pouco aplicadas independentemente do tamanho da empresa.

Contribuições metodológicas / sociais / gerenciais: Esse estudo indica um caminho a ser seguido pelas pequenas empresas para seu crescimento gerencial, bem como demonstra que o setor da construção civil necessita ampliar a utilização de ferramentas gerenciais como uma alternativa para melhorar o gerenciamento e aumentar o desempenho das construções.

Originalidade / relevância: Esse artigo se diferencia por comprovar estatisticamente os resultados apresentados e por contribuir para ampliar o conhecimento quanto às ferramentas gerenciais utilizadas no setor da construção civil.

Palavras-chave: Construção civil. Gestão da construção. Ferramentas gerenciais.

Este é um artigo publicado em acesso aberto (Open Access) sob a licença Creative Commons Attribution, que permite uso, distribuição e reprodução em qualquer meio, sem restrições desde que o trabalho original seja corretamente citado. 


\begin{abstract}
Purpose: The objective of this work is to identify which management tools differentiate micro and small enterprises from medium and large organizations, as well as to assess the level of use of these tools, considering the size of organizations.
\end{abstract}

Method / approach: The research was based on a Survey, which, through a questionnaire, collected data from 89 civil construction companies located in the Metropolitan Region of Curitiba. A comparison was made, through discriminant analysis and descriptive analysis of the collected data, regarding the use of management techniques between micro and small companies and medium and large organizations.

Main findings: The results showed that the certification of the Brazilian Program for Quality and Productivity of Habitat and the $A B C$ costing system are the methods that most differentiate large and medium organizations from micro and small companies. They also demonstrated that construction management tools are little applied regardless of the size of the company.

Methodological / social / managerial contributions: This study indicates a path to be followed by small companies for their managerial growth, as well as demonstrates that the civil construction sector needs to expand the use of managerial tools as an alternative to improve management and increase performance of buildings.

Originality / relevance: This article differs in that it statistically proves the results presented and contributes to expanding knowledge about the management tools used in the civil construction sector.

Keywords: Civil construction. Construction management. Management ferramentas.

\title{
1 INTRODUÇÃO
}

Devido às crises financeiras que ocorrem por todo o mundo, é cada vez mais importante que as organizações do setor da construção pratiquem uma melhor gestão de suas obras. Para isso, as empresas precisam ter conhecimento sobre as práticas de gerenciamento mais utilizadas nas organizações, bem como aquelas que melhor se adaptam a cada uma delas (Tereso et al., 2019).

A adoção de sistemas de controle de um projeto permite o alcance das metas financeiras e de prazo (Abdul-Rahman et al., 2006). Uma das possíveis formas de se realizar esse controle é adotando o uso de métodos de gerenciamento, uma vez que são capazes de produzir resultados satisfatórios de desempenho em projetos de edificação (Patah \& Carvalho, 2016). Segundo Trojanowska e Dostatni (2017), existem muitos métodos descritos na literatura capazes de fornecer apoio ao gerenciamento de projetos de construção.

Observa-se que em relação aos processos gerenciais, o setor da construção civil é descrito pelo seu conservadorismo (Krainer et al., 2013). Nascimento e Santos (2003) afirmam que o segmento da construção é um dos menos desenvolvidos e mais tradicionais, ao mesmo tempo em que os projetos de construção são caracterizados por sua complexidade, incerteza e diversidade de atividades (Wu et al., 2017).

No país, conforme dados da Câmara Brasileira da Indústria da Construção (CBIC, 2019), a indústria da construção civil é também caracterizada em maioria por micro e pequenas empresas (MPEs), as quais são significativas na contribuição econômica e geração de 
empregos do setor. Apesar disso, as organizações desse segmento apresentam dificuldades em sua gestão empresarial, o que ocasiona um alto índice de mortalidade precoce dessas companhias (Santini et al., 2015).

Também se verifica que, enquanto as MPEs são reconhecidas por poucas camadas de gerenciamento e uma administração centralizada, na qual muitas vezes o proprietário opera o estabelecimento, as médias e grandes empresas (MGEs) possuem uma estrutura hierárquica com várias camadas de gestão, um estilo mais participativo de gerenciamento e a utilização frequente de mão de obra terceirizada (Poshdar et al., 2019). No entanto, ambos grupos se assemelham pela necessidade de inovação para melhorar suas perspectivas de negócio (Handayani \& Handoyo, 2020).

Dentro desse contexto, ainda que se encontre na bibliografia trabalhos sobre a estrutura organizacional de diferentes tamanhos de empresas, poucas pesquisas são encontradas quando se busca analisar os métodos de gerenciamento utilizados nas construções brasileiras. No entanto, esses estudos já foram realizados em diferentes países (Delgado-Hernandez et al. 2017; El-Sawalhi \& Enshassi, 2012; Sarhan et al., 2017; Singh \& Kumar, 2020). Portanto, buscando preencher essa lacuna, o objetivo deste artigo é identificar quais ferramentas gerenciais diferenciam as MPEs das MGEs, bem como avaliar o nível de utilização dessas ferramentas, levando em consideração o tamanho das organizações.

Sabendo-se que as ferramentas de gestão são um pré-requisito para permanecer competitivo no mercado da construção (Tereso et al., 2019), a pesquisa a respeito do assunto torna possível que os stakeholders do setor, como empreiteiros, construtores, fornecedores, e engenheiros tenham uma visão da implementação de técnicas gerenciais na construção na região avaliada.

Adicionalmente, possibilita-se expandir a literatura sobre as ferramentas gerenciais no campo da gestão da construção e ampliar o conhecimento sobre as MPEs e MGEs desse setor, que é pouco investigado na bibliografia (Costa et al., 2020). Além disso, o estudo permite analisar o uso das técnicas gerenciais no Brasil e comparar com as pesquisas realizadas em outros países, aproximando a literatura sobre gestão da construção brasileira com outros locais do mundo.

Para realizar esse estudo, a estratégia de pesquisa adotada foi o survey, por meio da coleta de dados a respeito de organizações da construção situadas na região metropolitana de Curitiba. Essas informações foram analisadas com o uso de estatística multivariada, o que permitiu observar quais as técnicas gerenciais diferenciam as pequenas e grandes empresas, possibilitando analisar como esses diferentes grupos se comportam.

Quanto à organização, esse trabalho está esquematizado do seguinte modo: a próxima seção aborda os estudos recentes realizados a respeito das ferramentas gerenciais; a seção três apresenta a estratégia de pesquisa, contemplando todos os processos do Survey; a seção 4 descreve os resultados obtidos da amostra coletada, da análise discriminante e apresenta a análise descritiva junto com a discussão dos resultados; por fim, são expostas as conclusões a respeito desse trabalho.

\section{AS PRINCIPAIS FERRAMENTAS DE GESTÃO RELACIONADAS AO SETOR DA CONSTRUÇÃO}

Para que se pudesse definir e analisar as ferramentas de gestão, estudos recentes a respeito de seu uso serão apresentados a seguir. 
Análise da utilização das ferramentas gerenciais na construção civil entre MPEs e MGEs

Alguns métodos gerenciais, como o gráfico de Gantt, EAP e PERT/CPM, são considerados usualmente conhecidos no ramo da construção civil (El-Sawalhi \& Enshassi, 2012; Rounds \& Segner, 2011; Shahu et al., 2012). De acordo com Rounds e Segner (2011), o gráfico de Gantt foi inventado durante o início de 1900 e tem sido adotado por muitos anos na construção. No entanto, no ranking das técnicas usadas pelas empresas da indústria de construção britânica, as ferramentas relacionadas à programação de atividades, como Gráfico de Gantt e PERT/CPM, ficaram em quarto lugar no grau de utilização. Controle da qualidade, medição de desempenho e tecnologia foram os grupos que ficaram respectivamente em primeiro, segundo e terceiro lugar em relação a sua aplicação (Delgado-Hernandez \& Aspinwall, 2005).

El-Sawalhi e Enshassi (2012) Em seu estudo efetuado na Faixa de Gaza, verificaram que $44 \%$ da população do estudo 'nunca' usou a ferramenta EAP, enquanto apenas $17 \%$ 'sempre' usou EAP. O baixo uso da EAP pode ser atribuído ao desconhecimento de sua importância e ao desconhecimento de sua utilidade. Quando questionados sobre o agendamento de atividades de construção, metade dos entrevistados (51\%) usavam "frequentemente" gráficos de Gantt. Segundo os pesquisadores, o gráfico de Gantt é uma ferramenta amplamente utilizada devido à sua simplicidade e aplicabilidade em todas as fases de uma obra.

A programação CPM existe desde 1950 e embora seja considerada uma ferramenta básica de controle de projeto que é comumente praticada em todos os projetos de construção, os resultados da pesquisa realizada por Galloway (2006) demonstram que a programação CPM ainda não é um requisito obrigatório nem um controle de projeto que conquistou a confiança da indústria da construção. No survey realizado, apenas $47,6 \%$ dos respondentes indicaram que o agendamento CPM é sempre obrigatório em seus projetos.

Uma outra ferramenta gerencial estudada nos últimos anos é o Custeio Baseado em Atividades $(A B C)$, exercida principalmente na gestão financeira das empresas (Nascimento Neto, 2002). Apesar da preocupação das empresas em relação a eficiência do uso dos recursos, o uso dessa ferramenta não é uma realidade nas empresas de construção civil (Souza \& Mello, 2011). Em pesquisa realizada por Nascimento Neto (2002), nota-se que de maneira geral, existe pouca taxa de adoção das empresas, devido ao sistema utilizado pelas companhias atenderem suas necessidades, a sua complexidade de implantação e uso e o seu custo-benefício. Quanto à pesquisa de Innes et al. (2000), o Sistema ABC de custeio mostrouse mais presente em grandes organizações e mostrou seu decréscimo de utilização entre empresas do Reino Unido entre os anos de 1994 e 1999.

Outra técnica de gestão de obras, o benchmarking, permite que os dados de medição de desempenho, os quais não eram amplamente identificados e coletados nas empresas de construção (Costa et al., 2006) passem a ser conhecidos. Segundo survey feito por Rigby e Bilodeau (2005), o benchmarking é a segunda ferramenta mais implementada entre empresas de diferentes setores nas regiões da América do Norte, América Latina e Europa.

Métodos de gestão mais recentes, como os sistemas integrados, possibilitados pelo uso de ferramentas como o Enterprise Resource Planning (ERP), também têm ganhado espaço no mercado progressivamente (Chung et al., 2009). Apesar da necessidade de custo e tempo para sua implantação (Hadidi et al., 2017), a pesquisa realizada por Krainer et al. (2013), mostra que empresas com ERP são mais desenvolvidas comparadas às que não utilizam a ferramenta.

Ainda, quanto aos métodos recentes, é importante destacar os aplicados na filosofia lean, como o Kaizen, o Percentual de Planejamento Concluído (PPC), o PDCA e o 5S, entre 
vários outros existentes. O estudo realizado por Sarhan et al. (2017) em 282 empresas de construção na Arábia Saudita, mostra que não existem significativas diferenças entre grandes, pequenas e médias empresas, exceto as ferramentas de melhoria de segurança da obra. De modo geral, esse estudo concluiu que embora as ferramentas lean aumentem a produtividade e diminuam os desperdícios do canteiro, as técnicas não são amplamente praticadas no país e a adoção das técnicas é de maneira prolongada. Forbes e Ahmed (2010), justificam isso pelo fato de a construção civil ser resistente à mudança. Corroborando com esses resultados, os estudos de Johansen e Walter (2007) demonstram que $76 \%$ das empresas pesquisadas não empregam nenhum ou apenas um dos conceitos de gerenciamento da filosofia lean na indústria da construção alemã. Outra ferramenta dentro da filosofia lean, o PPC mostrou-se efetivo para medir a produtividade dos empreendimentos (Angelim et al., 2020).

Bajjou e Chafi (2018) em uma análise das respostas relacionadas à implementação de técnicas lean no Marrocos, mostraram que 39\% dos entrevistados não estão familiarizados com nenhum sistema enxuto de práticas de construção e nunca ouviram falar delas, 35\% estão familiarizados com as práticas, mas ainda não as executam em seus projetos e $26 \%$ estão familiarizados com as técnicas lean e as estão executando em seus projetos. (Bajjou \& Chafi, 2018).

Mais especificamente sobre o sistema 5S, conforme Marhani et al. (2018), a ferramenta $5 \mathrm{~S}$ é uma das mais aplicadas para a redução dos resíduos e uma das mais apropriadas para atingir o desempenho esperado do projeto, em pesquisa executada na indústria da construção na Malásia. Resultado semelhante é encontrado por Singh e Kumar (2020), no qual o método $5 S$ e os métodos de controle visual (como painéis de exibição) apareceram como os mais utilizados como ferramentas de gerenciamento visual na indústria de construção da Índia. No entanto, em pesquisa feita por Johansen e Walter (2007), nas organizações de construção da Alemanha, apenas 16\% dos canteiros de obras utilizam o processo 5 S.

Sobre o uso da ferramenta PDCA nas construções, poucos trabalhos sobre a frequência de seu uso nas construções são encontrados, apesar de sua extensa aplicabilidade e funcionalidade dentro dos canteiros de obras (Ren et al., 2015).

Por fim, quanto à qualidade das construções, a principal norma adotada pelas construtoras brasileiras é o Programa Brasileiro da Qualidade e Produtividade do Habitat (PBQP-H), cujo objetivo é o aumento da qualidade e a modernização produtiva do setor (Nercolini, 2019). De acordo com os dados do Ministério do Desenvolvimento Regional (2021), há 166 empresas com essa certificação no estado do Paraná, e de acordo com dados do Sindicato da Indústria da Construção Civil no Estado do Paraná (Sinduscon-PR, 2021), há 624 estabelecimentos de construção cadastrados no sindicato. Portanto, estima-se que aproximadamente $26,60 \%$ das empresas possuem essa certificação no estado. No entanto, mesmo certificadas, as empresas da construção possuem dificuldades na manutenção da certificação. Entre as maiores dificuldades, cita-se o gerenciamento de resíduos, o controle de estoque, a segurança no trabalho e a compatibilização de projetos (Araújo \& Rivelini, 2016).

Assim sendo, seguindo os estudos encontrados a respeito dos métodos de gestão, foram definidas as ferramentas estudadas nessa pesquisa, que estão conceitualmente apresentadas de forma sucinta na Tabela 1. 
Tabela 1

Ferramentas de gestão usadas na construção civil.

\begin{tabular}{cl}
\hline Método & Conceito \\
\hline Ciclo PDCA & $\begin{array}{l}\text { É uma ferramenta de gerenciamento que orienta a sequência de atividades. Os quatro } \\
\text { principais princípios são: Planejamento, executar, verificar e atuar (Ren et al., 2015). }\end{array}$ \\
\hline Sistema de & $\begin{array}{l}\text { Método de organização, mobilização e transformação de organizações. São cinco níveis que } \\
\text { ajudam na diminuição de recursos desnecessários (Sarhan et al., 2017). }\end{array}$ \\
\hline Pusteio ABC & $\begin{array}{l}\text { Permite a melhor visualização dos custos por meio da análise das atividades realizadas na } \\
\text { empresa e suas relações com os níveis de custos (Souza \& Mello, 2011). }\end{array}$ \\
\hline ERP & $\begin{array}{l}\text { Sistemas de informações que funcionam na forma de software e aumentam o o } \\
\text { compartilhamento de informações e integridade dos processos (Hadidi et al., 2017). }\end{array}$ \\
\hline PBQP-H & $\begin{array}{l}\text { Certificação nacional baseada na norma ISO 9001, aplicável às empresas da construção civil, } \\
\text { que possui uma série de requisitos com objetivo de melhorar a qualidade dos habitats (Araújo } \\
\text { \& Rivelini, 2016). }\end{array}$ \\
\hline Kaizen & $\begin{array}{l}\text { Kaizen significa “mudar para melhor". Não é considerado uma ferramenta específica, mas sim } \\
\text { a constante e geral otimização em etapas (Sarhan et al., 2017). }\end{array}$ \\
\hline EAP & $\begin{array}{l}\text { Técnica que possibilita gerenciar o escopo do projeto. É a divisão do projeto em componentes } \\
\text { pequenos e mais fáceis de gerenciar (El-Sawalhi \& Enshassi, 2012). }\end{array}$ \\
\hline Gráfico de & $\begin{array}{l}\text { Ferramenta que mostra as atividades de um projeto e a duração da atividade em ordem de } \\
\text { execução (Delgado-Hernandez et al., 2017). }\end{array}$ \\
\hline Pantt & $\begin{array}{l}\text { É a relação entre a quantidade de tarefas realizadas e às previstas, sendo capaz de fornecer o } \\
\text { nível de confiabilidade dos planos de trabalho (Angelim et al., 2020). }\end{array}$ \\
\hline Benchmarking & $\begin{array}{l}\text { Processo sistemático de comparação de desempenho de uma empresa em relação a outras } \\
\text { semelhantes, visando o aumento da performance (Costa et al., 2006). }\end{array}$ \\
\hline $\begin{array}{l}\text { A rede PERT/CPM é representada por um diagrama de redes, que permite visualizar o inter- } \\
\text { relacionamentos das atividades do projeto (Delgado-Hernandez \& Aspinwall, 2005). }\end{array}$ \\
\hline
\end{tabular}

\section{ESTRATÉGIA DE PESQUISA}

A metodologia aplicada nesta pesquisa foi o Survey e o instrumento de pesquisa comumente utilizado é o questionário, que pode assumir diferentes características e escalas (Hair et al., 2009).

\subsection{PLANEJAMENTO DO SURVEY}

O planejamento do Survey iniciou-se pela determinação das variáveis. As variáveis delimitadas para esse trabalho foram baseadas nos estudos apresentados anteriormente, nos quais foram determinados quais os métodos de gestão seriam estudados. Essas variáveis foram apresentadas na Tabela 1, totalizando 11 variáveis independentes.

Para fazer a análise entre os diferentes tamanhos das organizações, usou-se como base a classificação do Serviço Brasileiro de Apoio às Micro e Pequenas Empresas e Departamento Intersindical de Estatística e Estudos Socioeconômicos (SEBRAE \& DIESSE, 2018). A partir dessa classificação, criou-se a variável de categórica (dependente) pelo tamanho das empresas, que foi dividida em dois grupos: o grupo 1 corresponde às microempresas e empresas de pequeno porte (com até 49 funcionários) enquanto o grupo 2 corresponde às empresas de médio porte e grandes empresas (mais que 49 funcionários).

A população estabelecida para o Survey foi construtoras localizadas na Região Metropolitana de Curitiba, portanto a amostra foram empresas localizadas nesta área. A Região Metropolitana de Curitiba é a que possui o maior número de construtoras no estado do Paraná segundo o Sinduscon-PR (2021). Em 2019, a cidade de Curitiba possuía em funcionamento 3691 empresas do setor da Construção Civil. Isso representa 19,6\% das empresas de construção atuantes em todo o Estado do Paraná (CBIC, 2019). 
O método de amostragem adotado foi o não probabilístico por conveniência, ou seja, as empresas da amostra foram selecionadas pela maior disponibilidade e facilidade de obter informações necessárias ao estudo. Isso se deve ao grande número de construtoras existentes na região da população e da dificuldade de obter dados a respeito de todas as empresas atuantes do mercado, bem como da indisponibilidade de algumas organizações.

O instrumento de coleta de dados escolhido para o estudo foi o questionário, dividido em duas partes principais: a parte de identificação do perfil da organização e do entrevistado e a parte dos métodos de gestão de obras usados pelas empresas. A parte de identificação da empresa possui questões discursivas e de múltipla escolha, como nome da empresa, ano de fundação, cidade, número de funcionários e forma de administração da empresa. Além disso, foram abordadas questões relacionadas ao perfil do entrevistado, como o cargo e área de atuação na organização.

A segunda parte do formulário continha as questões relacionadas ao uso dos métodos de gestão. Para a mensuração do uso das ferramentas, usou-se a escala tipo Likert com sete pontos. A escala é bidirecional e crescente, onde a ferramenta poderia variar de inexistente até altamente desenvolvida.

\subsection{COLETA DE DADOS}

Antes de iniciar efetivamente a coleta de dados foi realizado um teste piloto em 15 empresas da população estudada, com o objetivo de aprimorar a clareza, objetividade e a viabilidade das questões e dos conceitos abordados. As questões foram ajustadas e foi realizado a coleta de dados finais.

Aproximadamente 100 construtoras foram previamente contatadas e convidadas a responder o questionário, dessas 74 retornaram o questionário. No total, com o teste piloto, a amostra foi constituída de 89 empresas. Conforme Hair et al. (2009), o tamanho mínimo recomendado para a amostra é de cinco observações por variável independente para realizar a análise multivariada. Portanto, nessa pesquisa seriam necessárias 55 observações, o que torna o tamanho da amostra suficiente.

A coleta dos dados foi realizada de dezembro de 2019 até junho de 2020. Os contatos de telefone ou e-mail das empresas foram obtidos por meio de seu website. Inicialmente, os questionários eram entregues pessoalmente aos entrevistados. Posteriormente, foi realizado o envio eletrônico do formulário, por meio da ferramenta Formulários Google.

\subsection{ANÁLISE DOS DADOS}

As análises dos dados foram realizadas em três partes: a primeira parte diz respeito a caracterização da amostra, na segunda parte foi feita a análise discriminante e na terceira parte foi executada a análise descritiva.

A análise discriminante é uma técnica estatística multivariada aplicada quando a variável dependente é categórica, com o objetivo de discriminar e classificar diferentes grupos (Hair et al., 2009). No estudo, a variável categórica se refere ao tamanho das empresas, de forma a dividir a amostram em dois grupos: as MPEs e as MGEs. Para os dois segmentos foram analisadas as variáveis independentes, que são as ferramentas gerenciais definidas para a pesquisa com base no referencial bibliográfico, apresentadas na Tabela 1.

Com o objetivo de observar o comportamento de cada método individualmente, foi utilizada a estatística descritiva por meio de histogramas, de modo a aprofundar o entendimento sobre as diferenças e semelhanças encontradas em cada técnica gerencial em 
Análise da utilização das ferramentas gerenciais na construção civil entre MPEs e MGEs

relação ao tamanho das organizações. Foi utilizado o software Microsoft Office Excel 2019 para o armazenamento dos dados e para a caracterização da amostra e o software Statistical Package for the Social Sciences (SPSS) para a realização da análise discriminante e da análise descritiva.

\section{ANÁLISE E DISCUSSÃO DOS RESULTADOS}

\section{1 CARACTERIZAÇÃO DA AMOSTRA}

A Tabela 2 apresenta os principais elementos que caracterizam a amostra estudada.

Tabela 2

Características da amostra coletada

\begin{tabular}{|c|c|c|c|}
\hline Característica observada & & Número & Percentual \\
\hline \multirow{3}{*}{ Tipo de administração } & Profissional & 45 & $51 \%$ \\
\hline & Familiar & 33 & $37 \%$ \\
\hline & Mista & 11 & $12 \%$ \\
\hline \multirow{7}{*}{ Tipo de sociedade } & Sociedade Limitada & 64 & $72 \%$ \\
\hline & Sociedade anônima fechada & 7 & $9 \%$ \\
\hline & Empresa pública & 4 & $4 \%$ \\
\hline & Sociedade anônima aberta & 3 & $3 \%$ \\
\hline & Sociedade de economia mista & 2 & $2 \%$ \\
\hline & Empresa individual & 2 & $2 \%$ \\
\hline & Outro & 7 & $8 \%$ \\
\hline \multirow{4}{*}{ Tamanho das empresas } & Microempresa & 19 & $21 \%$ \\
\hline & Empresa de Pequeno Porte & 34 & $38 \%$ \\
\hline & Empresa de médio porte & 12 & $14 \%$ \\
\hline & Grande empresa & 24 & $27 \%$ \\
\hline \multirow{5}{*}{$\begin{array}{c}\text { Área de atuação do } \\
\text { entrevistado }\end{array}$} & Gerenciamento de obras & 56 & $63 \%$ \\
\hline & Projetos & 24 & $27 \%$ \\
\hline & Orçamento & 5 & $6 \%$ \\
\hline & Suprimentos & 2 & $2 \%$ \\
\hline & Outra & 2 & $2 \%$ \\
\hline \multirow{4}{*}{$\begin{array}{l}\text { Cargo ocupado pelo } \\
\text { entrevistado }\end{array}$} & Engenheiro ou arquiteto & 51 & $57 \%$ \\
\hline & Estagiário & 19 & $21 \%$ \\
\hline & Diretor ou gerente & 14 & $16 \%$ \\
\hline & Técnico em edificações & 5 & $6 \%$ \\
\hline
\end{tabular}

Destaca-se que a maior parte das empresas possuem a administração profissional (51\%) e são de sociedade limitada (72\%). Quanto ao tamanho das empresas, observa-se que o grupo formado pelas micro e pequenas empresas corresponde a $59 \%$ da amostra (53 organizações), enquanto o grupo constituído pelas empresas de médio porte e grandes empresas se refere a $41 \%$ da amostra (36 organizações). Quanto ao cargo ocupado pelos respondentes, a maior parte são engenheiros ou arquitetos (57\%), depois tem-se estagiários (21\%) e diretores ou gerentes (16\%). A principal área de atuação dos entrevistados é gerenciamento de obras (63\%) seguida pela área de projetos $(27 \%)$.

\subsection{ANÁLISE DISCRIMINANTE}

A análise discriminante das ferramentas gerenciais foi executada utilizando-se o tamanho da empresa como fator de discriminação. Foram estabelecidos dois grupos: o grupo 1 são as micro e pequenas empresas e o grupo 2 são as médias e grandes organizações. 
Inicialmente é feita uma série de testes para validar os resultados estatísticos alcançados. Primeiro a análise do $\mathrm{M}$ de Box, presente na Tabela 3.

Tabela 3

Resultado do teste $M$ de Box.

M de Box

\begin{tabular}{l|lr}
\hline$Z$ & Aprox. & 81,701 \\
\hline & df1 & 1,060 \\
& df2 & 66 \\
& Sig. & 17014,402 \\
& &, 347 \\
\hline
\end{tabular}

A análise do $M$ de Box foi realizada para verificar a igualdade das matrizes de covariância entre os grupos (Hair et al., 2009). Se o valor encontrado estiver acima do nível de significância de 5\%, não se rejeita a hipótese nula e a igualdade das matrizes possui sustentação, ou seja, o pressuposto do teste é atendido. Na Tabela 3 verifica-se que a significância é de $0,347(>0,05)$, logo pode se dar prosseguimento às análises.

Outro teste realizado, a estatística de U, também chamada de Lambda de Wilk's, varia de 0 a 1 e indica a variabilidade de médias entre os grupos. Esse teste baseia-se na hipótese nula de que as médias populacionais dos dois grupos são iguais. Nesse caso, espera-se rejeitar a hipótese nula, para que a função discriminante seja considerada significativa. Conforme a Tabela 4, a significância está abaixo dos $5 \%(0,018<0,05)$, portanto considera-se que o modelo estudado é estatisticamente discriminante.

Tabela 4

Resultado do Lambda de Wilk's

\begin{tabular}{c|c|c|c|c}
\hline Teste de funções & Lambda de Wilks & Qui-quadrado & $\mathrm{df}$ & Sig. \\
\hline 1 &, 755 & 22,958 & 11 &, 018 \\
\hline
\end{tabular}

O Lambda de Wilk's também pode ser efetuado para cada variável independente, para se ter uma prévia da capacidade discriminante das variáveis, utilizando-se para isso o teste de igualdade entre as médias de grupo. Quanto mais próxima de zero o teste de significância da variável, maior a probabilidade de se rejeitar a hipótese nula de que as médias dos grupos são iguais. Assim sendo, segundo o resultado apresentado na Tabela 5, as variáveis "PBQP-H" e "sistema de custeio $A B C$ " são as únicas que rejeitam a hipótese nula de que as médias dos grupos são iguais, pois seus valores não ultrapassam o nível de significância de 0,05. Logo, são as variáveis que apresentam maior poder discriminante.

Tabela 5

Testes de igualdade de médias de grupo.

\begin{tabular}{lccccc}
\hline & Lambda de Wilks & Z & df1 & df2 & Sig. \\
\hline EAP &, 987 & 1,118 & 1 & 87 &, 293 \\
GRÁFICO DE GANTT &, 978 & 1,991 & 1 & 87 &, 162 \\
PPC & 1,000 &, 005 & 1 & 87 &, 944 \\
SISTEMA DE CUSTEIO ABC &, 967 & 3,001 & 1 & 87 &, 047 \\
REDE PERT/COM &, 994 &, 493 & 1 & 87 &, 485 \\
PBQP-H &, 868 & 13,253 & 1 & 87 &, 000 \\
5S &, 992 &, 665 & 1 & 87 &, 417 \\
ERP &, 982 & 1,572 & 1 & 87 &, 213 \\
KAIZEN &, 999 &, 083 & 1 & 87 &, 774 \\
BENCHMARKING &, 999 &, 097 & 1 & 87 &, 756 \\
PDCA &, 991 &, 826 & 1 & 87 &, 366 \\
\hline
\end{tabular}


Na Tabela 6, o resultado da matriz de estruturas contém quais são as variáveis efetivamente consideradas discriminantes entre os grupos. De acordo com Hair et al. (2009), o valor da função da variável deve estar acima de 0,40 para que essa seja considerada um fator de diferenciação entre os grupos.

Nota-se que as variáveis "PBQP-H" e "sistema de custeio $A B C$ " atingiram esse valor, desta maneira são as ferramentas gerenciais discriminantes entre os dois tamanhos de empresas considerados. Observa-se que esse resultado é compatível com o encontrado no teste igualdade de médias dos grupos, o que torna a análise consistente. As outras variáveis não podem ser classificadas como discriminantes, devido ao baixo valor de sua função. No entanto, o baixíssimo valor apresentado pelas variáveis "benchmarking", "kaizen" e "PPC", indicam que essas são possivelmente ferramentas com características semelhantes entre os dois grupos.

Tabela 6

Matriz de estruturas

\begin{tabular}{lc}
\hline Variável & Função \\
\hline PBQP-H &, 684 \\
SISTEMA DE CUSTEIO ABC &, 426 \\
GRÁFICO DE GANTT &, 265 \\
ERP &, 236 \\
EAP &, 199 \\
PDCA &, 171 \\
5S &, 153 \\
REDE PERT/COM &, 132 \\
BENCHMARKING &, 059 \\
KAIZEN &, 054 \\
PPC &, 013 \\
\hline
\end{tabular}

Na parte final da análise, tem-se a estatística de classificação, na qual mostra o quanto de acerto o modelo estatístico teve na classificação das variáveis. Analisando seu resultado na Tabela 7, nota-se que $73,0 \%$ dos casos foram classificados corretamente. Do grupo 1 , que são as micro e pequenas empresas, $83 \%$ dos casos foram classificados corretamente e $58 \%$ dos casos das médias e grandes empresas.

Tabela 7

Resultados da classificação

\begin{tabular}{c|lcccc}
\hline \multicolumn{2}{c}{ Tamanho } & & 1,0 & 2,0 & \multicolumn{2}{c}{ Total } \\
\hline \multirow{4}{*}{ Original } & Contagem & 1,0 & 44 & 9 & 53 \\
& & 2,0 & 15 & 21 & 36 \\
\cline { 2 - 6 } & $\%$ & 1,0 & 83,0 & 17,0 & 100,0 \\
& & 2,0 & 41,7 & 58,3 & 100,0 \\
\hline
\end{tabular}

a. $73,0 \%$ de casos agrupados originais classificados corretamente.

\subsection{ANÁLISE DESCRITIVA}

A análise discriminante aponta quais são as variáveis discriminantes entre os grupos, contudo não indica quais são exatamente as diferenças entre eles. Então, para aprofundar o entendimento do comportamento dessas variáveis, fez-se a análise descritiva dos dados por meio de seus histogramas.

A primeira variável analisada é a mais discriminante entre os grupos, "PBQP-H". No seu histograma, apresentado na Figura 1 é notável que a diferença entre as MPEs e as MGEs se 
dá pelo maior atendimento da norma pelo segundo grupo. Conforme Maciel et al. (2019), muitos gestores de pequenas empresas da construção enxergam a certificação PBQP-H como um custo e não como uma ferramenta capaz de auxiliar o gerenciamento da obra. Ainda, segundo Nercolini (2019), algumas construtoras consideram o processo de certificação burocrático e pouco produtivo. Nesse sentido, mesmo o PBQP-H sendo benéfico para a gestão organizacional das construtoras (Nercolini, 2019), a certificação parece não ser uma prioridade para as empresas, principalmente às micro e pequenas. De acordo com Araújo e Rivelini (2016), há uma dificuldade geral por parte dos gestores para implantar e manter o PBQP-H em pleno funcionamento.

O maior nível de atendimento por parte das grandes empresas também se deve a exigência da certificação para acessos a financiamentos governamentais, como o programa Minha Casa Minha Vida (Duarte et al., 2020). Assim sendo, esse estudo demonstra estatisticamente a baixa adesão do PBQP-H pelas pequenas empresas. Considerando que essas empresas são uma parcela significativa do mercado da construção, questiona-se se a aplicabilidade de certificação não deveria ser expandida também a esse grupo, de modo a possibilitar a melhoria no seu sistema de gestão.

Figura 1

Histograma das variáveis "PBQP-H" e "sistema de custeio $A B C$ ".

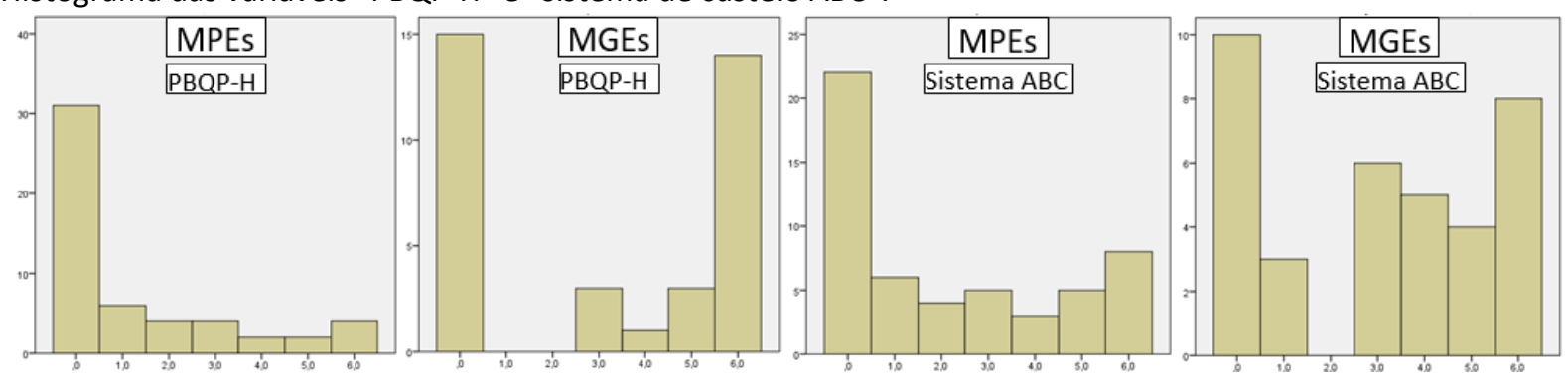

A segunda variável considerada discriminante foi o sistema de custeio $A B C$, que observando os histogramas presentes na Figura 1, também é mais presente nas médias e grandes organizações do que nas micro e pequenas empresas. Esse resultado é similar ao encontrado por Innes et al. (2000), no qual seu uso, no Reino Unido, é mais frequente nas maiores organizações. Quanto à pesquisa realizada por Nascimento Neto (2002), que mostra uma baixa taxa de adoção dessa ferramenta nas indústrias brasileiras, o resultado é parcialmente coincidente, visto que o seu uso depende do tamanho da organização.

As pequenas empresas muitas vezes não possuem uma gestão financeira eficiente (Santini et al., 2015), o que pode explicar o pouco uso dessa ferramenta por essas empresas. Por isso, a implementação do sistema de custeio ABC pelas MPEs pode ser um modo eficiente de auxiliar no gerenciamento financeiro dessas organizações, visto que é uma prática já executada nas maiores empresas. Além de que, diferentemente do PBQP-H, a aplicação desse método não depende de nenhuma certificação, sendo necessária apenas o conhecimento sobre seu funcionamento, o que torna essa ferramenta acessível a todas as empresas.

As variáveis presentes na Figura 2 são as que não são consideradas discriminantes, porém ainda obtiveram um valor significativo na matriz de estruturas da análise multivariada. Destaca-se inicialmente o alto grau de não utilização das ferramentas de gestão, tanto pelas grandes quanto pelas pequenas empresas. De modo geral, os métodos gerenciais não parecem ser difundidos nas organizações do estudo. Apesar disso, algumas diferenças são encontradas. Os métodos EAP, gráfico de Gantt e PDCA, apresentaram maior diferença de 
utilização, sendo mais desenvolvidos pelas grandes empresas. Essas ferramentas são consideradas tradicionais no setor da construção (El-Sawalhi \& Enshassi, 2012; Rounds \& Segner, 2011), portanto esperava-se que seu uso fosse significativo em todas as empresas, independentemente de seu tamanho.

Esse resultado é coerente com os de Delgado-Hernandez e Aspinwall (2005) e demonstram a necessidade de se ampliar o uso dos métodos gerenciais, que apesar de conhecidos no setor da construção, não apresentaram alta aplicabilidade. Outro fator a ser observado na Figura 2 é que, apesar de uma das metodologias implantadas pela certificação do PBQP-H ser o PDCA (Pereira \& Gonzalez, 2017), a utilização do PDCA é inferior à aplicação da certificação pelas médias e grandes empresas, o que é um possível indício de ineficiência do PBQP-H. Isso porque esse percentual deveria ser no mínimo igual, senão superior, já que o uso do PDCA não está necessariamente atrelado à certificação.

Figura 2

Histograma das variáveis classificadas como não discriminantes.

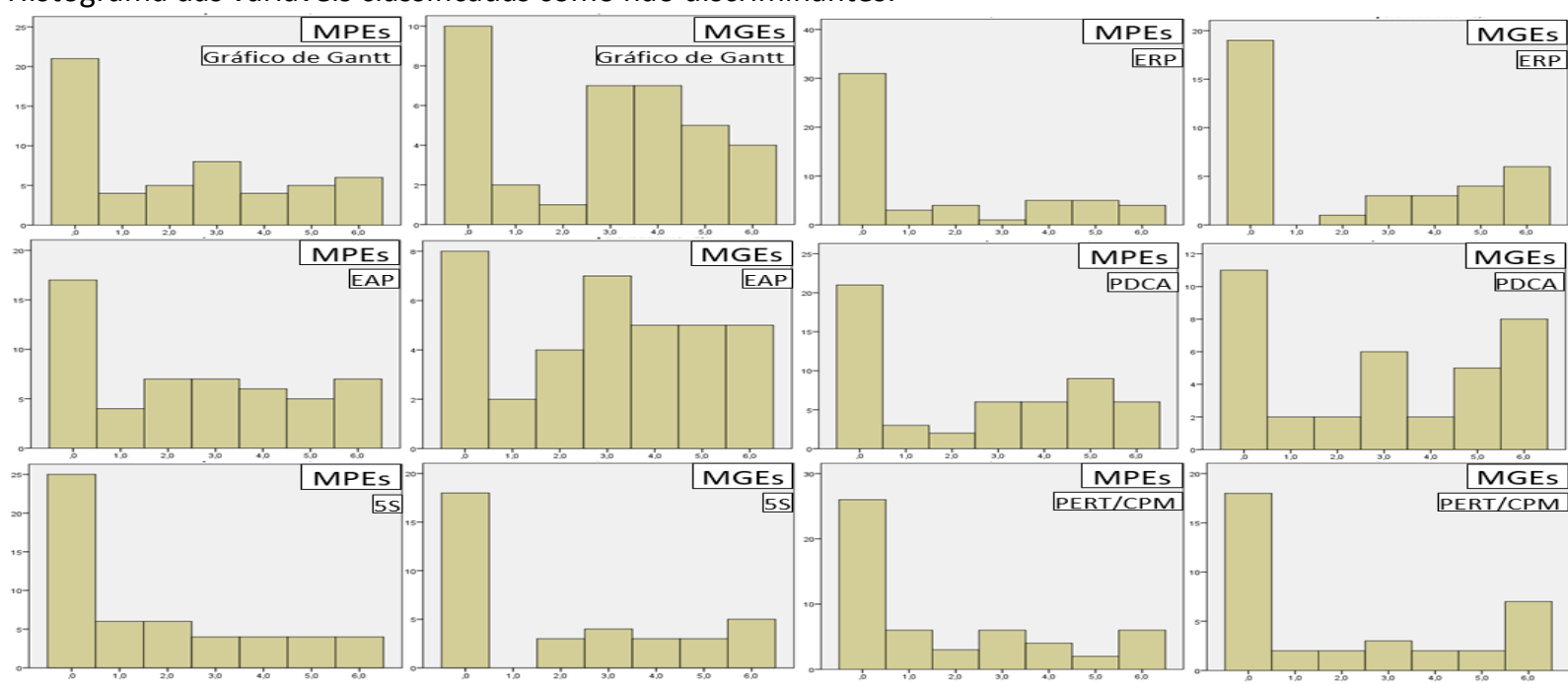

Os métodos 5S, rede PERT/CPM e ERP apresentaram baixo grau de utilização independentemente do tamanho da empresa. Realizando um comparativo com os estudos de Johansen e Walter (2007), esse resultado é próximo ao encontrado nas empresas de construção da Alemanha, onde o processo $5 \mathrm{~S}$ também se mostrou pouco usado. No entanto, é diferente do encontrado na indústria da construção da Malásia, por Marhani et al. (2018) e na Índia por Singh e Kumar (2020), países nos quais o 5S é constantemente aplicado.

O PERT/CPM, que também é uma ferramenta tradicional no meio da construção (Shahu et al., 2012), apresentou resultado próximo ao encontrado por Galloway (2006), no qual o método não é predominantemente empregado na indústria da construção dos Estados Unidos. Entre as razões para sua pouca utilização, cita-se o fato da ferramenta ter alta necessidade de lógica, necessitar de trabalho excessivo e ter dependência de especialistas (Galloway, 2006).

Quanto ao sistema ERP, ainda que Chung et al. (2009) indique o aumento de implementação desse sistema pelas grandes organizações, os resultados mostram seu baixo nível de uso por todas as empresas. Entretanto, observa-se que esse método apresenta uma distribuição uniforme nas pequenas organizações, enquanto nas médias e grandes organizações sua distribuição está crescente à direita. Isso significa que as maiores empresas tendem a usar mais o método, ainda que de forma muito discreta. 
O planejamento de recursos empresariais (ERP) é visto como um investimento caro para empresas de arquitetura e construção, que demanda tempo e uma equipe para operacionalizar o sistema (Hadidi et al., 2017), os quais são possíveis motivos para sua baixa implementação nas empresas da região.

Por fim, as três últimas variáveis se referem aos métodos com menor valor na matriz de estruturas da análise discriminante, ou seja, os que possivelmente são mais semelhantes. Essas ferramentas gerenciais são benchmarking, kaizen e PPC. Constata-se pela Figura 3 que as técnicas apresentaram resultados muito próximos para ambos os grupos. Em comum, o alto percentual de respostas concentradas na não implementação das três ferramentas para todos os tamanhos de empresa, com baixo percentual de pleno desenvolvimento das ferramentas nos canteiros de obra.

As ferramentas utilizadas na filosofia lean, como Kaizen, PPC e o processo 5S, possuem resultados próximos aos estudos realizados por Sarhan et al. (2017), Johansen e Walter (2007) e Bajjou e Chafi (2018) visto que, apesar do conhecimento da existência desses métodos, são pouco adotadas nas obras. Esse resultado demonstra que a filosofia Lean Construction provavelmente é pouco empregada nas construções da região. De acordo com Sarhan et al. (2018), as principais barreiras enfrentadas na aplicação de práticas lean são as influências das práticas tradicionais, a cultura organizacional desfavorável, a falta de habilidades técnicas e conhecimento sobre as ferramentas lean.

Figura 3

Histograma das variáveis classificadas como semelhantes.

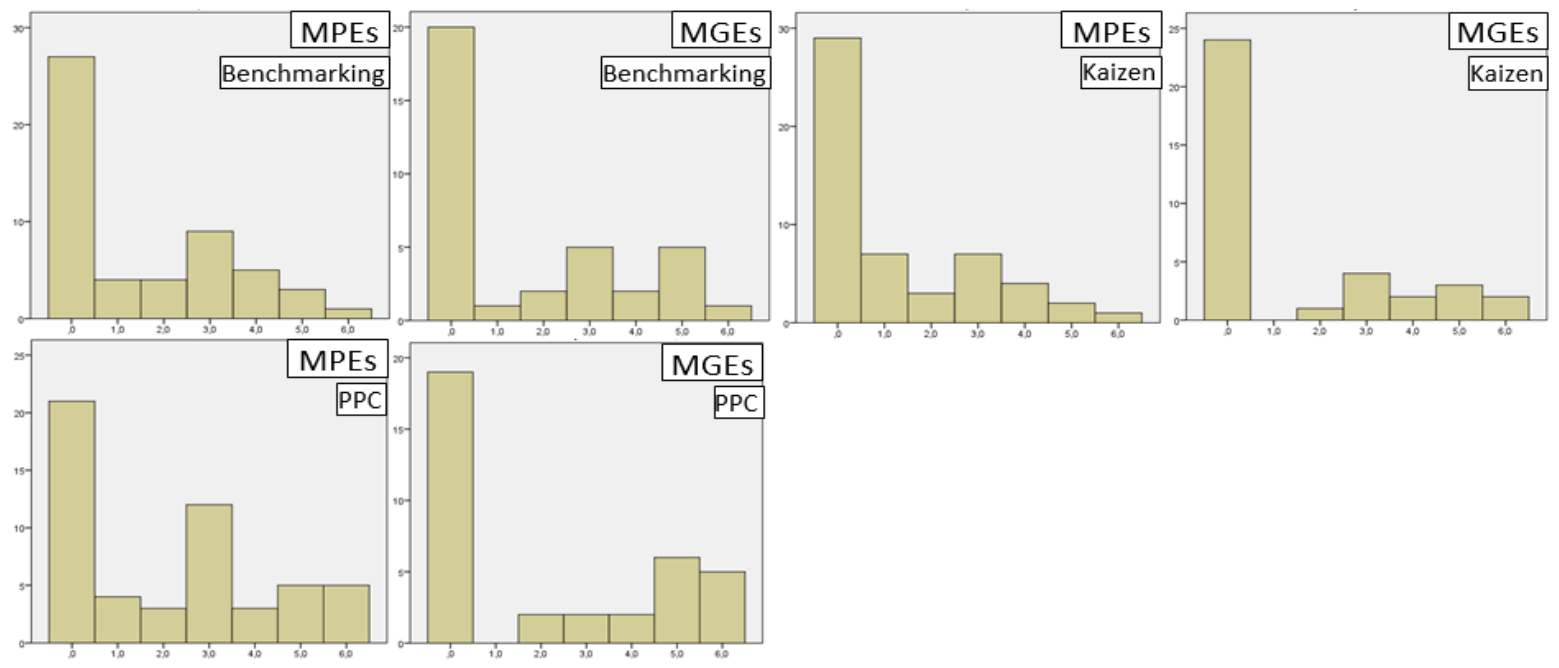

Por fim, a técnica do benchmarking mostra-se dissemelhante aos estudos de Rigby e Bilodeau (2005), pois tem um baixo percentual de utilização. Também se destaca seu uso ligeiramente maior em pequenas empresas do que nas grandes. Ainda, cabe salientar que muitas vezes o benchmarking é realizado de forma empírica pelos gestores da empresa, de forma precária e sem o devido planejamento e registro da aplicação da técnica (Lima \& Oliveira, 2018). Isso pode ser confirmado pelo seu histograma, uma vez que as respostas referentes ao nível 6, de total desenvolvimento do benchmarking, apresentam a menor quantidade de respostas. 
Análise da utilização das ferramentas gerenciais na construção civil entre MPEs e MGEs

\section{CONSIDERAÇÕES FINAIS}

Neste artigo foi realizada uma análise discriminante das ferramentas gerenciais entre as micro e pequenas empresas e médias e grandes organizações da construção civil. Nessa análise, o certificado do PBQP-H e o sistema de custeio $A B C$ foram as técnicas que diferenciaram esses dois grupos de empresas. Pela análise descritiva, foi possível verificar que as grandes empresas possuem a certificação, bem como realizam mais o seu gerenciamento financeiro por meio do sistema de custeio $A B C$.

Portanto, essas duas ferramentas são pontos cruciais, pois mostram em quais aspectos as grandes empresas estão mais desenvolvidas quanto ao uso de ferramentas de gestão. Desta forma, esse estudo indica que as pequenas construções da região devem aperfeiçoar principalmente sua gestão de qualidade e de custos dos seus canteiros de obras visando seu crescimento.

No entanto, o que se destaca nessa pesquisa é o baixo percentual de implementação da maior parte das ferramentas gerenciais, inclusive aquelas já consideradas tradicionais no meio da construção civil, tanto para as PMEs quanto para as MGEs. Nesse sentido, fica evidente o baixo investimento das empresas da construção civil em técnicas de gestão.

Esse resultado auxilia no entendimento das falhas gerenciais nas obras, que causam atrasos e perda de produtividade e consequentemente, lucratividade. Nesse contexto, esse estudo sugere que o setor da região invista na ampliação do uso das ferramentas gerenciais, como uma das formas de melhorar a eficiência na gestão dos canteiros. Para que isso seja possível, o conhecimento dos métodos deve estar inserido dentro do meio acadêmico, como por exemplo, nas universidades, e deve chegar também aos gestores de obras, seja através da capacitação profissional, realizada pela empresa ou por investimentos por meio de políticas públicas.

Sendo assim, esse processo de expansão da aplicação das ferramentas de gestão é capaz de produzir uma maior padronização de procedimentos e facilitar o atendimento das metas estabelecidas, tornando o ambiente da construção civil um local propício à instalação de novas técnicas gerenciais, além de reduzir seu caráter conservador.

Quanto as limitações do estudo, destaca-se que esse estudo foi realizado em uma região específica do país, portanto seu resultado pode ser diferente dependendo do local onde é aplicado. Nesse sentido, sugere-se que essa mesma pesquisa seja realizada em outros locais, para que seja possível realizar comparações e para que se compreenda qual o contexto do gerenciamento dos canteiros de obra em outras regiões do país.

Sugere-se também a utilização de métodos probabilísticos de amostragem e o aumento do tamanho da amostra, permitindo a utilização de análises estatísticas mais robustas. Assim, aumenta-se a confiabilidade do estudo, diminuindo seu caráter exploratório e tornando-o mais conclusivo sobre a utilização das ferramentas gerenciais na região.

\section{REFERÊNCIAS}

Araújo, J. C., \& Rivelini, A. R. B. (2016). PBQP-H: dificuldades na gestão da qualidade, segundo os representantes da direção. Revista UNINGÁ Review, 28(3), 78-84.

Abdul-Rahman, H., Berawi, M. A., Berawi, A. R., Mohamed, O., Othman, M., \& Yahya, I. A. (2006). Delay mitigation in the Malaysian construction industry. Journal of Construction Engineering and Management, 132(2), 125-133.

https://doi.org/10.1061/(ASCE)0733-9364(2006)132:2(125).

RGO - Revista Gestão Organizacional, Chapecó, v. 15, n. 1, p. 132-149, jan./abr., 2022. 
Angelim, V. L., Alves, T. D. C. L., Lima, M. M. X. D., \& Barros Neto, J. D. P. (2020). Planejamento de médio prazo: panorama de sua aplicação na construção civil. Ambiente Construído, 20(1), 87-104. https://doi.org/10.1590/s167886212020000100364.

Bajjou, M. S., \& Chafi, A. (2018). Lean construction implementation in the Moroccan construction industry. Journal of Engineering, Design and Technology, 16(4), 533-556. https://doi.org/10.1108/JEDT-02-2018-0031.

Câmara Brasileira da Indústria da Construção Civil - CBIC. (2019). Número de estabelecimentos e tamanho (por empregados ativos na construção civil) - Brasil e Regiões. http://www.cbicdados.com.br/menu/empresas-deconstrucao/estabelecimentos-na-construcao.

Chung, B., Skibniewski, M. J., \& Kwak, Y. H. (2009). Developing ERP systems success model for the construction industry. Journal of Construction Engineering and Management, 135(3), 207-216. https://doi.org/10.1061/(ASCE)07339364(2009)135:3(207).

Costa, D. B., Formoso, C. T., Kagioglou, M., Alarcón, L. F., \& Caldas, C. H. (2006). Benchmarking initiatives in the construction industry: lessons learned and improvement opportunities. Journal of Management in Engineering, 22(4), 158-167. https://doi.org/10.1061/(ASCE)0742-597X(2006)22:4(158).

Costa, G. R., Sousa, G. C. M. \& Cândido, L. P. (2020). Fatores críticos de sucesso: a percepção de gestores de MPE de construção no semiárido nordestino. Encontro Nacional de Tecnologia do Ambiente Construído, Porto Alegre, RS, Brasil. https://entac2020.com.br/anais-2020/artigos/196.pdf.

Delgado-Hernandez, D. J., \& Aspinwall, E. M. (2005). Improvement tools in the UK construction industry. Construction Management and Economics, 23(9), 965-977. https://doi.org/10.1080/01446190500204705.

Delgado-Hernandez, D. J., Cruz-Cruz, C. C., \& Vences-García, P. Y. (2017). Improvement management tools in the construction industry: case study of Mexico. Journal of Construction Engineering and Management, 143(4), 05016024. https://doi.org/10.1061/(ASCE)CO.1943-7862.0001229.

Duarte, P. B. M., Branco, R. B. C., \& do Espírito Santo, K. N. A. (2020). Gestão da qualidade na construção civil: uma análise do programa Brasileiro de qualidade e produtividade no habitat (PBQP-H) e da ISO 9001. Brazilian Journal of Development, 6(3), 14817-14827. https://doi.org/10.34117/bjdv6n3-376.

El-Sawalhi, N. I., \& Enshassi, A. (2012). Application of project time management tools and techniques to the construction industry in the Gaza Strip. Construction Economics and Building, 5(1). https://doi.org/10.5130/ajceb.v5i1.2937 . 
Análise da utilização das ferramentas gerenciais na construção civil entre MPEs e MGEs

Forbes, L. H., \& Ahmed, S. M. (2010). Modern construction: lean project delivery and integrated practices. New York: Taylor \& Francis Group.

Galloway, P. D. (2006). Survey of the construction industry relative to the use of CPM scheduling for construction projects. Journal of Construction Engineering and Management, 132(7), 697-711. https://doi.org/10.1061/(asce)07339364(2006)132:7(697).

Hadidi, L., Assaf, S., \& Alkhiami, A. (2017). A systematic approach for ERP implementation in the construction industry. Journal of Civil Engineering and Management, 23(5), 594603. https://doi.org/10.3846/13923730.2016.1215348.

Hair, J. F., Black, W. C. Babin, B. J., Anderson, R. E., \& Tatham, R. L. (2009). Análise Multivariada de dados (6a ed.). Porto alegre: Bookman.

Handayani, R., \& Handoyo, R. D. (2020). Better Performance Prospect of Large-Medium Enterprises: The Role of Innovation. Journal of Economics, Business, \& Accountancy Ventura, 22(3), 411-423. https://doi.org/10.14414/jebav.v22i3.2041.

Innes, J., Mitchell, F., \& Sinclair, D. (2000). Activity-based costing in the UK's largest companies: a comparison of 1994 and 1999 survey results. Management Accounting Research, 11(3), 349-362. https://doi.org/10.1006/mare.2000.0135.

Johansen, E., \& Walter, L. (2007). Lean construction: Prospects for the German construction industry. Lean Construction Journal, 3(1), 19-32.

Krainer, C. W. M., Krainer, J. A., larozinski Neto, A., \& Romano, C. A. (2013). Análise do impacto da implantação de sistemas ERP nas características organizacionais das empresas de construção civil. Ambiente Construído, 13(3), 117-135. https://doi.org/10.1590/S1678-86212013000300008.

Lima, Á. R., \& Oliveira, A. S. (2018). O processo de benchmarking: um estudo em empresas de construção civil no município de João Pessoa. Escritos Contables y de Administración, 9(2), 13-35.

Maciel, F. W. F., Cândido, L. F., \& Rocha, V. F. T. (2019). PBQP-H e Norma de Desempenho: um estudo de múltiplos casos com micro e pequenas construtoras cearenses. Simpósio Brasileiro de Gestão e Economia da Construção e Encuentro Latino-americano de Gestión y Economia de La Construcción, Londrina, PR, Brasil. https://eventos.antac.org.br/index.php/sibragec/article/view/55.

Marhani, M. A., Bari, N. A. A., Ahmad, K., \& Jaapar, A. (2018). The implementation of lean construction tools: Findings from a qualitative study. Chemical Engineering Transactions, 63, 295-300. https://doi.org/10.3303/CET1863050.

Ministério do Desenvolvimento Regional. (2021). Sistema de avaliação da conformidade de serviços e obras - SiAC. http://pbqp-h.mdr.gov.br/projetos_siac_empresas.php. 
Nascimento Neto, R. V. (2002). Utilização do sistema de custeio ABC por indústrias brasileiras: uma pesquisa empírica. Congresso Brasileiro de Custos, São Paulo, SP, Brasil. https://anaiscbc.emnuvens.com.br/anais/issue/archive.

Nascimento, L. A., \& Santos, E. T. (2003). A indústria da construção na era da informação. Ambiente Construído, 3(1), 69-81.

Nercolini, R. V. (2019). Avaliação do impacto da transição da certificação ISO 9001: 2015 e o não alinhamento com PBQP-H SiAC: 2017 na estratégia organizacional em construtoras de edificações de Curitiba. [Dissertação de Mestrado, Universidade Tecnológica Federal do Paraná]. http://repositorio.utfpr.edu.br/jspui/handle/1/4198.

Patah, L. A., \& Carvalho, M. M. D. (2016). Sucesso a partir de investimento em metodologias de gestão de projetos. Production, 26(1), 129-144. https://doi.org/10.1590/01036513.048312 .

Pereira, M. A., \& Gonzalez, E. F. (2017). Avaliação de serviços controlados pelo PBQPH. Revista UNINGÁ Review, 32(1), 242-254.

Poshdar, M., Gonzalez, V. A., Antunes, R., Ghodrati, N., Katebi, M., Valasiuk, S., Alqudah H., \& Talebi, S. (2019). Diffusion of lean construction in small to medium-sized enterprises of housing sector. Proceedings of the Annual Conference of the International. Group for Lean Construction (IGLC), Dublin, Irlanda. https://doi.org/10.24928/2019/0257.

Ren, M. M., Ling, N., Wei, X., \& Fan, S. H. (2015). The application of PDCA cycle management in project management. Proceedings of the International Conference on Computer Science and Applications, Wuhan, Hubei, China. https://ieeexplore.ieee.org/abstract/document/7810878/.

Rigby, D., \& Bilodeau, B. (2005). The Bain 2005 management tool survey. Strategy \& Leadership, 33(4), 4-12. https://doi.org/10.1108/10878570510607997.

Rounds, J. L., \& Segner, R. O. (2011). Construction supervision. New Jersey: John Wiley \& Sons.

Santini, S., Favarin, E. V., Nogueira, M. A., de Oliveira, M. L., \& Ruppenthal, J. E. (2015). Fatores de mortalidade em micro e pequenas empresas: um estudo na região central do Rio Grande do Sul. Revista Eletrônica de Estratégia \& Negócios, 8(1), 145-169.

Sarhan, J. G., Xia, B., Fawzia, S., \& Karim, A. (2017). Lean construction implementation in the Saudi Arabian construction industry. Construction Economics and Building, 17(1), 4669. https://doi.org/10.5130/AJCEB.v17i1.5098.

Sarhan, J., Xia, B., Fawzia, S., Karim, A., \& Olanipekun, A. (2018). Barriers to implementing lean construction practices in the Kingdom of Saudi Arabia (KSA) construction industry. Construction Innovation, 18(2), 246-272. https://doi.org/.10.1108/ci-04-2017-0033. 
Análise da utilização das ferramentas gerenciais na construção civil entre MPEs e MGEs

Shahu, R., Pundir, A. K., \& Ganapathy, L. (2012). An Empirical Study on Flexibility: A Critical Success Factor of Construction Projects. Global Journal of Flexible Systems Management, 13(3), 123-128. https://doi.org/10.1007/s40171-012-0014-5.

Serviço Brasileiro de Apoio às Micro e Pequenas Empresas \& Departamento Intersindical de Estatística e Estudos Socioeconômicos. (2018). Anuário do Trabalho nos Pequenos Negócios. São Paulo. https://www.dieese.org.br/anuario/201.

Sindicato da Indústria da Construção Civil no Estado do Paraná. (2021). Construtoras Associadas. https://sindusconpr.com.br/nossos-associados.

Singh, S., \& Kumar, K. (2020). A study of lean construction and visual management tools through cluster analysis. Ain Shams Engineering Journal, 12(1), 1153-1162. https://doi.org/10.1016/j.asej.2020.04.019.

Souza, A. A., \& Mello, E. (2011). Análise da cadeia de valor: um estudo no âmbito da gestão estratégica de custos de empresas da construção civil da grande Porto Alegre. Revista Contemporânea de Contabilidade, 8(15), 11-39. https://doi.org/10.5007/21758069.2011v8n15p11.

Tereso, A., Ribeiro, P., Fernandes, G., Loureiro, I., \& Ferreira, M. (2019). Project management practices in private organizations. Project Management Journal, 50(1), 6-22. https://doi.org/10.1177/8756972818810966.

Trojanowska, J., \& Dostatni, E. (2017). Application of the theory of constraints for project management. Management and Production Engineering Review, 8(3), 87-95.

Wu, G., Liu, C., Zhao, X., \& Zuo, J. (2017). Investigating the relationship between communication-conflict interaction and project success among construction project teams. International Journal of Project Management, 35(8), 1466-1482. 